\title{
Heating of the auroral ionosphere by traveling ionospheric disturbances initiated by atmospheric gravity waves
}

\author{
Syam Sundar De ${ }^{1, \star}$, Bijoy Bandyopadhyay ${ }^{1}$, Suman Paul ${ }^{1}$, Dilip Kumar Haldar ${ }^{1}$, Mridul Bose ${ }^{2}$ \\ ${ }^{1}$ Centre of Advanced Study in Radio Physics and Electronics, University of Calcutta, Kolkata, India \\ ${ }^{2}$ Department of Physics, Jadavpur University, Kolkata, India
}

\author{
Article history \\ Received September 26, 2009; accepted March 18, 2010. \\ Subject classification: \\ Ionospheric dynamics, Plasma physics, Auroral phenomena
}

\begin{abstract}
In the presence of perturbations of the thermospheric auroral region produced by traveling ionospheric disturbances during the propagation of atmospheric gravity waves, an analytical expression of the velocity of the thermospheric plasma is derived through magnetohydrodynamic formalism. The expressions of the Joule heating and the viscous heating are derived, and their rates of variation are presented. A threshold height for their transition has been determined from their ratio, which is in agreement with the experimental data. The analysis indicates that the time taken by the thermospheric plasma to reach a steady-state corresponds to the nature of the traveling ionospheric disturbances in the medium.
\end{abstract}

\section{Introduction}

Ionospheric convection modulated by solar wind and magnetohydrodynamic wave coupling to the dayside magnetosphere produce atmospheric gravity waves (AGWs) [Hocke and Schlegel 1996, Kirchengast 1996]. In the auroral zone, AGWs are associated with the auroral electrojet [Millward et al. 1993, Prölss and Roemar 2000, Yuan et al. 2005]. These ionospherically forced AGWs deposit their momentum and energy in the upper and lower atmosphere, thereby providing a link between the solar wind and the Earth atmosphere.

Coupling of the magnetosphere to the ionosphere is much felt through the precipitation of energetic particles that promote large amounts of local ionization. Joule dissipation of these currents is a major ionospheric energy source at auroral latitudes, which appears to be several times greater than that directly associated with particle precipitation.

Traveling ionospheric disturbances (TIDs) are frequently observed at high and middle latitudes, and they can be taken as manifestations of middle-scale ionospheric irregularities arising as a response to AGWs [Oliver et al. 1994, Bristow et al. 1996, Hernandez-Pajares et al. 2006, Nicolls and Heinselman 2007, Onishi et al. 2009]. During geomagnetic storms, the atmosphere at high latitudes is greatly affected by the energy deposited in the auroral region. Auroral processes, e.g., auroral electric currents and charge-particle precipitation, produce Joule heating and gravity waves that introduce TIDs [Hocke and Schlegel 1996, Afraimovich et al. 2000]. The characteristics of TIDs change with latitude, longitude, local time, season, and solar cycle [Hernandez-Pajares et al. 2006, Kotake et al. 2006]. Currently, different investigations are being carried out to explore the geographical and temporal variabilities of the observed gravity waves, and their relationships with the observed TIDs, so that the upward energy flow from the troposphere to the mesosphere, through the stratosphere, can be understood. This way of energy coupling from the lower to the upper atmosphere, along with the resulting ionospheric effects, contributes to the upper atmospheric energy balance [Kersey and Hughes 1989, Allen and Vincent 1995; Fritts and Alexander 2003].

Auroral heating of the neutral atmosphere is a part of the more general question of the total energy budget. There are large-scale modeling studies of the responses of the neutral thermosphere to ion convection. The local responses of the neutral thermospheric temperature and mechanism for Joule and particle heating have been modeled. The propagation of gravity waves is mainly in the E-region of the ionosphere by Joule heating and Lorentz forcing.

The propagation of AGWs in the neutral atmosphere and their ionospheric signatures (i.e., TIDs) have been studied both experimentally and theoretically for many years. On the basis of these theoretical and experimental investigations, several models have been developed to describe the main characteristics of the AGW-TID relationships on a global scale. The models reflect our level of understanding of the basic physical principles governing 


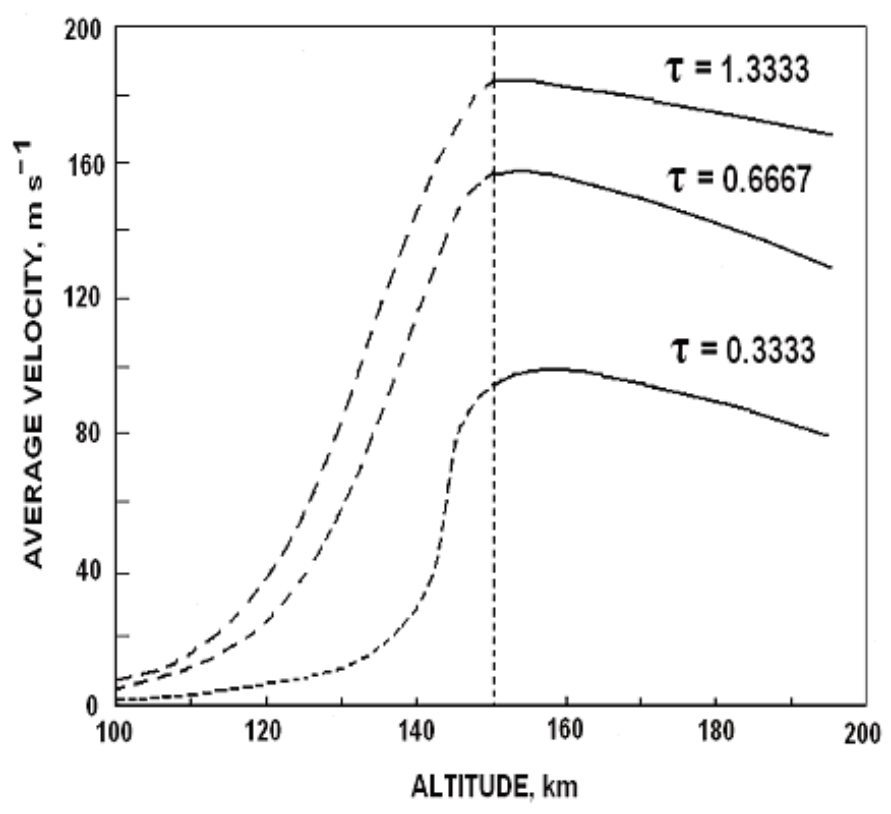

Figure 1. Variations in the average velocity of the thermospheric plasma with altitude at different characteristic times $(\tau)$. The different curves show that the thermospheric plasma approaches greater velocities with increasing $\tau$, to reach a steady-state at different altitudes. The dashed lines are for functional completeness (these values are not physically valid).

the atmospheric and ionospheric processes. A comprehensive simulation of the relationships between all of the fundamental parameters of the neutral atmosphere perturbed by AGWs and all of the fundamental ionospheric parameters was performed by Kirchengast [1996], for comparisons with incoherent scatter data.

Under the stated physical situation, the auroral region of the ionosphere can be characterized by different nonlinear processes that arise from variations in the velocity distribution of the thermospheric constituents, medium temperature, ionizing frequency, effective collision frequency, and recombination coefficients of electrons and ions [Schlegel 1982, Prölss 2006].

Investigations into the perturbations in temperatures and wind in the auroral region of the upper atmosphere have indicated the presence of high latitude heat sources [Istomin and Leyser 1997]. Some studies have suggested that energy transfer by precipitating particles provides the dominant contributions [Allan and Cook 1974, Olson and Moe 1974], while Joule dissipation and ion drag can take on more important roles, particularly surrounding the auroral oval and within the polar cap [Cole 1975]. The non-linear energy transfer from electromagnetic radiation from the auroral magnetospheric zone to the down-flowing electrons is directly associated with the transient auroral disturbances [Hanasz et al. 2006, Zesta et al. 2006].

Auroral electric currents and charged-particle precipitation produce Joule heating, gravity waves and traveling ionospheric disturbances that can initiate temperature increases and temperature fluctuations [Fagundes et al. 1996, Belikovich et al. 1997]. The presence of a fluctuating electric field initiates Joule heating, along with viscous heating [Brekke and Kamide 1996]. Magnetosphereionosphere coupling mechanisms also provide information about Joule heating rates [Yuan et al. 2005], along with various other electrodynamic parameters. This heating provides the largest contribution to the total energy budget in the medium.

Gravity waves that propagate to thermospheric heights and interact with the auroral ionospheric plasma give rise to TIDs. Knowledge of the intensity of the wave disturbances of the neutral atmosphere and of the ionospheric parameters are important for radio forecasting, and aeroplane and space-craft navigation. To date, TIDs have had a beneficial impact on field users of the global navigation satellite system [Coster and Tsugawa 2008].

An analytical expression of the upper thermospheric plasma-flow velocity in the auroral region is derived here through magnetohydrodynamic formalism.

The influence of ionization and recombination processes, of density and temperature fluctuations of the thermospheric medium, and of gravity and viscosity are taken into account in this analysis.

The spatial distribution of the velocity and its altitude dependence within the auroral zone are explored. The expressions of Joule heating and viscous heating are derived. For different characteristic times, the variations in the velocity with the altitude are investigated. The data are presented here and discussed.

The height-dependent ratio of the viscous heating to the Joule heating rate terms in generating AGWs in the auroral electrojets at $100 \mathrm{~km}$ to $150 \mathrm{~km}$ altitudes is derived and the results are compared with those of a previous study [Yuan et al. 2005].

\section{Mathematical formulations}

The physical situation can be represented by the following momentum balance, continuity and state equations:

$$
\begin{gathered}
\rho \frac{\partial \vec{v}}{\partial t}+\rho(\vec{v} \cdot \nabla) \vec{v}+\rho v \vec{v}-\rho \vec{g}= \\
=-\nabla p-\vec{J} \times \vec{B}+\mu \nabla^{2} \vec{v}+\frac{1}{3} \mu \nabla(\nabla \cdot \vec{v}) \\
\frac{\partial \rho}{\partial t}+\nabla \cdot(\rho \vec{v})=q-\alpha \rho^{2} \\
p=\rho k_{B} T
\end{gathered}
$$

where $\rho$ is the mass density, $\nu$ the effective electron-neutral molecule collision frequency, $\mu$ the coefficient of viscosity of the medium, $q$ the rate of ionization, $\alpha$ the electron-ion recombination coefficient, $\vec{B}$ the geomagnetic field vector, and $\vec{B} \times \vec{J}$ the source term. $\vec{J}$ is the current density, which is 
chosen according to:

$\vec{J}=\left(\sigma_{0}-\sigma_{1}\right)(\vec{E} \cdot \hat{k}) \hat{k}+\sigma_{1}(\vec{E}+\vec{v} \times \vec{B})+\sigma_{2}(\vec{E} \times \hat{k})$

where $\vec{E}$ is the auroral electric-field vector, $\sigma_{0}, \sigma_{1}$ and $\sigma_{2}$ are the longitudinal, Pedersen and Hall conductivities, respectively, and the other symbols have their standard representations. For the present purposes, the current density has been chosen in a form of a Gaussian distribution, which is specifically in a plane perpendicular to the direction of flow. For simplicity of the computational work, the time development is taken as a function with a rapid build-up, a steady phase, and a subsequent decay. Fluctuations in the mass densities and mean temperatures are taken as $\rho=\rho_{0}(1$ $+\eta)$ and $\mathrm{T}=\mathrm{T}_{0}(1+\theta)$, where $\eta$ is the fluctuation in mass densities and $\theta$ is the fluctuation in mean temperatures.

When combined with the perturbations, Equation (1) can be expressed as:

$$
\frac{\partial^{2} v}{\partial x^{2}}+\frac{\partial^{2} v}{\partial z^{2}}-a \frac{\partial v}{\partial t}-b v=c
$$

where,

$$
\begin{gathered}
a=\frac{\rho_{0}(1+\eta)}{\mu} \\
b=\frac{v \rho_{0}(1+\eta)+\sigma_{1} B_{x} B_{y}}{\mu} \\
c=\frac{k_{B} \rho_{0} T_{0}}{\mu} \frac{\partial \theta}{\partial x}+\frac{\sigma_{1} E_{y} B_{z}-\sigma_{0} E_{z} B_{y}}{\mu}-\frac{\rho_{0}(1+\eta)}{\mu} g
\end{gathered}
$$

The plasma is considered to be bounded within the auroral region $-L$ to $+L$ along the $x$-direction, where the momentum can be transferred to the surrounding region $(|x|>L)$ through the action of a viscous force. This is used to treat the analysis within a finite domain. Thus, the initial and boundary conditions are specified as $v(x, z, 0)=0$, $v(-L, z, 0)=v(+L, z, 0) \neq 0$, and $\vec{E}=0$ and $\sigma_{1}=0$ for $|x|>L$, with $x, z$ and $t$ as independent variables. The boundary condition is valid for all values of $t$, although for the mathematical simplification, this is chosen as $t=0$. The study covers the altitude range from $90 \mathrm{~km}$ to $200 \mathrm{~km}$.

The solution of Equation (5) can be expressed in the following propagatory form:

$$
\begin{aligned}
v(x, z, t) & =C_{1} \exp \left\{\alpha_{1}(k x+p z-\omega t)\right\}+ \\
& +C_{2} \exp \left\{\alpha_{2}(k x+p z-\omega t)\right\}-\frac{c}{b}
\end{aligned}
$$

where,

and

$$
\alpha_{1}=\frac{-a \omega+\sqrt{a^{2} \omega^{2}+4 b\left(k^{2}+p^{2}\right)}}{2\left(k^{2}+p^{2}\right)}
$$

$$
\alpha_{2}=\frac{-a \omega-\sqrt{a^{2} \omega^{2}+4 b\left(k^{2}+p^{2}\right)}}{2\left(k^{2}+p^{2}\right)}
$$

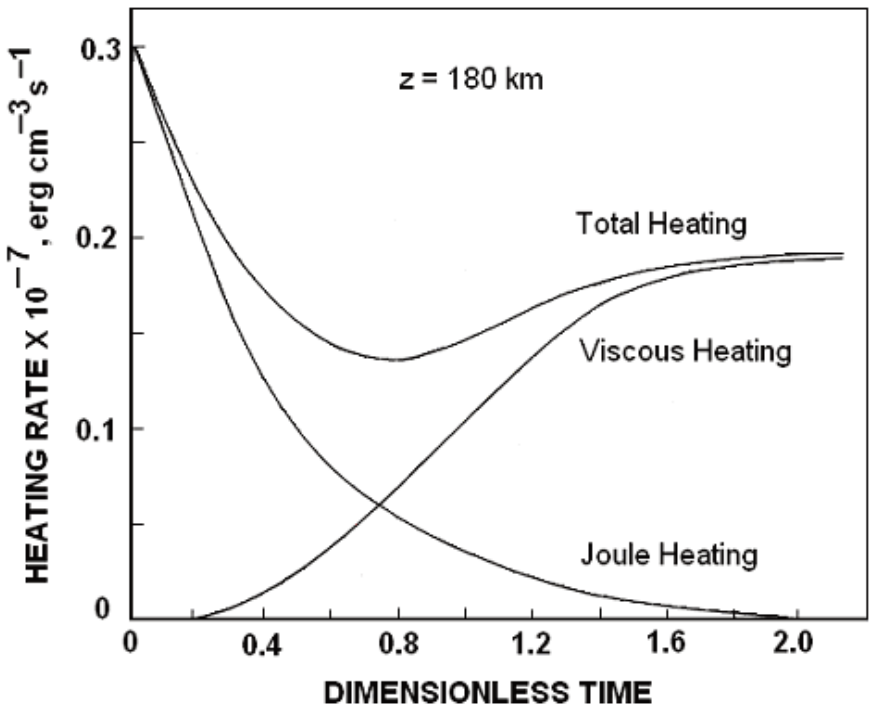

Figure 2. Changes in the heating rates with dimensionless time at an altitude $(\mathrm{z})$ of $180 \mathrm{~km}$. The total heating is the sum of the Joule heating and the viscous heating, which approaches a steady value.

are the roots of the auxiliary equation, and $C_{1}$ and $C_{2}$ are constants.

The time constant required for the thermospheric plasma flow to reach a steady-state is defined by the characteristic time, which is given by $\tau=\rho / \sigma_{1} B^{2}$. This parameter has been introduced to simplify the problem. Also, for the dimensionless time, this characteristic time is adopted to normalize the time. It is considered as $t / \tau=\delta$, where $\delta$ (a constant) is the dimensionless time. Thus, in terms of characteristic time, the solution for the average thermospheric plasma velocity yields:

$$
\begin{aligned}
\nu(x, z, \tau) & =C_{1} \exp \left\{\alpha_{1}(k x+p z-\omega \delta \tau)\right\}+ \\
& +C_{2} \exp \left\{\alpha_{2}(k x+p z-\omega \delta \tau)\right\}-\frac{c}{b}
\end{aligned}
$$

The solution of Equation (7) gives the Joule heating $\left(Q_{J}\right)$ and the viscous heating $\left(Q_{V}\right)$ through the following expressions:

$$
\begin{gathered}
Q_{J}=\sigma_{1} E_{x}^{2}+\sigma_{1} E_{y}^{2}+\sigma_{0} E_{z}^{2}+\sigma_{1}\left(B_{z} E_{x}-B_{x} E_{z}\right) \\
{\left[C_{1} \exp \left\{\alpha_{1}(k x+p z-\omega \delta \tau)\right\}+\right.} \\
\left.+C_{2} \exp \left\{\alpha_{2}(k x+p z-\omega \delta \tau)\right\}-\frac{c}{b}\right] \\
Q_{V}=\mu \nu\left(k^{2}+p^{2}\right)\left[C_{1} \alpha_{1}^{2} \exp \left\{\alpha_{1}(k x+p z-\omega \delta \tau)\right\}+\right. \\
\left.+C_{2} \alpha_{2}^{2} \exp \left\{\alpha_{2}(k x+p z-\omega \delta \tau)\right\}\right]
\end{gathered}
$$

The ratio of the viscous heating rate to the Joule heating rate terms can be readily obtained explicitly from these two expressions above, as: 


$$
\begin{aligned}
& R=\left|\frac{Q_{V}}{Q_{J}}\right| \\
& \mu \nu\left(k^{2}+p^{2}\right)\left[C_{1} \alpha_{1}^{2} \exp \left\{\alpha_{1}(k x+p z-\omega \delta \tau)\right\}+\right. \\
& \left.+C_{2} \alpha_{2}^{2} \exp \left\{\alpha_{2}(k x+p z-\omega \delta \tau)\right\}\right] \times \\
& \times\left[C_{1} \exp \left\{\alpha_{1}(k x+p z-\omega \delta \tau)\right\}+\right. \\
& +C_{2} \exp \left\{\alpha_{2}(k x+p z-\omega \delta \tau)\right\}- \\
& R=\frac{\left.-\frac{\left[k_{B} \rho_{0} T_{0} \frac{\partial \theta}{\partial x}+\sigma_{1} E_{y} B_{z}-\sigma_{0} E_{z} B_{y}-\rho_{0}(1+\eta) g\right]}{v \rho_{0}(1+\eta)+\sigma_{1} B_{x} B_{y}}\right]}{\sigma_{1} E_{x}^{2}+\sigma_{1} E_{y}^{2}+\sigma_{0} E_{z}^{2}+\sigma_{1}\left(B_{z} E_{x}-B_{x} E_{z}\right) \times} \\
& \times\left[C_{1} \exp \left\{\alpha_{1}(k x+p z-\omega \delta \tau)\right\}+\right. \\
& +C_{2} \exp \left\{\alpha_{2}(k x+p z-\omega \delta \tau)\right\}- \\
& \left.-\frac{\left[k_{B} \rho_{0} T_{0} \frac{\partial \theta}{\partial x}+\sigma_{1} E_{y} B_{z}-\sigma_{0} E_{z} B_{y}-\rho_{0}(1+\eta) g\right]}{\nu \rho_{0}(1+\eta)+\sigma_{1} B_{x} B_{y}}\right]
\end{aligned}
$$

The data of the Committee on Space Research International Reference Atmosphere (CIRA) and the International Reference Ionosphere (IRI) ionospheric models [Bilitza et al. 1993] are used in the computation. For an $80^{\circ} \mathrm{N}$ latitude at $180 \mathrm{~km}$ in altitude, the values of the electron densities, neutral particles, and ion and electron temperatures were taken from these models. FORTRAN subroutine programs were used to calculate the average velocity of the thermospheric plasma, the Joule heating and the viscous heating, and their ratio. The Dynamics Explorer (DE-2) satellite observations identified the enhancement as a storm phenomenon [Prölss 2006],

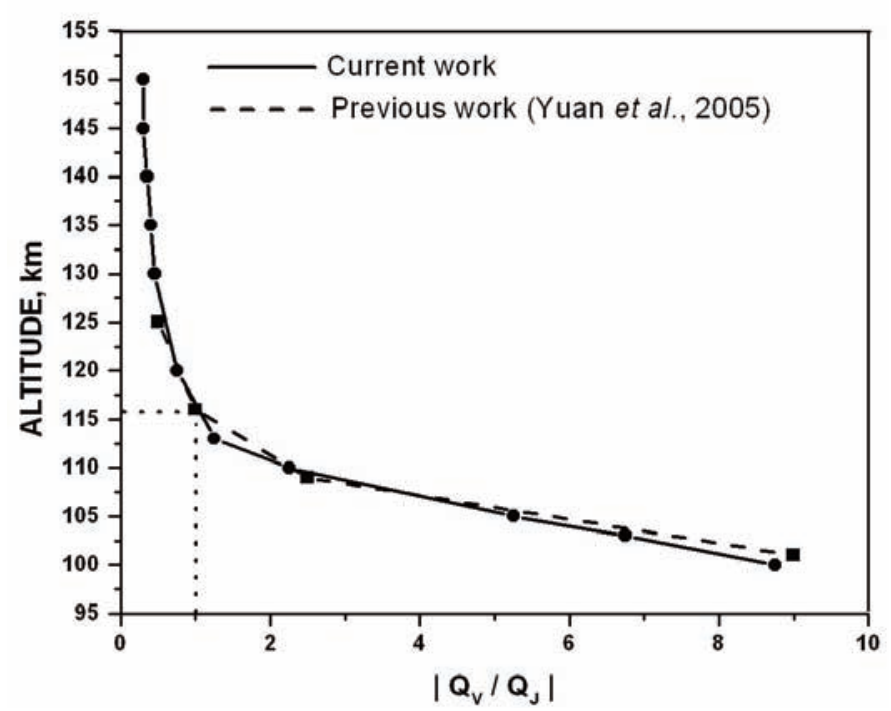

Figure 3. Variation of the ratio of the viscous heating rate to the Joule heating rate with altitude. The continuous curve connecting the filled circles represents the data from the present study, while the dashed curve joining the filled squares represents the data from the earlier study of Yuan et al. [2005]. which supported the results of the present study.

\section{Summary}

The variations in the average velocity of the thermospheric plasma flow with altitude for different characteristic times are shown in Figure 1. The different curves in Figure 1 show that the thermospheric plasma approaches higher velocities with increasing values of the characteristic time to reach a steady-state at different altitudes. The inclusion of the dashed lines in Figure 1 is for functional completeness: these values are not physically valid, as the Hall conductivity is negligible compared to the Pedersen conductivity in this region, although the Hall conductivity itself is not negligible.

Figure 2 shows the heating rate plotted against the dimensionless time at an altitude of $180 \mathrm{~km}$, as the occurrence of TIDs within the F-layer of the ionosphere almost maintains its peak levels around this region [Kirchengast 1996]. The average values of the Joule heating and the viscous heating in the disturbed volume are presented separately. The Joule heating is effective in the initial stages, while it decreases with time as the volume increases. When the motion is set up due to a dominating electric field, only then is there viscous heating. This increases due to the movement of the thermospheric plasma medium, and it eventually reaches a steady value.

Figure 3 shows the variations in the values for the ratio of the viscous heating to the Joule heating rate terms, $R$, derived from Equation (8), with the altitude ranging from $100 \mathrm{~km}$ to $150 \mathrm{~km}$. The present study is shown (Figure 3, continuous line) in comparison with a previous study (Figure 3, dotted line; Yuan et al. 2005), which was based on the European Incoherent Scatter (EISCAT) data during the period from 1989 to 1991 . According to this comparison, the method adopted in the present study is shown to be reasonable. Between $100 \mathrm{~km}$ and $125 \mathrm{~km}$ in altitude, the ratio ranges from 0.5 to 10 , while between $125 \mathrm{~km}$ and $150 \mathrm{~km}$, it is approximately constant, with a value of 0.3 . The viscous heating is relatively important below $116 \mathrm{~km}$, while the Joule heating dominates above this altitude. As Brekke and Kamide [1996] indicated, the relative importance of the two sources in generating the AGWs is critically dependent on the altitude. The present study supports this prediction quite satisfactorily.

Acknowledgements. The authors acknowledge with thanks the Indian Space Research Organization (ISRO), through S. K. Mitra Centre for Research in the Space Environment, University of Calcutta, Kolkata, India, for the financial support to carry out this study. The authors also thank the two reviewers for making important comments.

\section{References}

Afraimovich, E. L., E. A. Kosogorov, L. A. Leonovich, K. S. Palamarchouk, N. P. Perevalova and O. M. Pirog (2000). Determining parameters of large-scale traveling 
ionospheric disturbances of auroral origin using GPS arrays, J. Atmos. Sol. Terr. Phys., 62, 553-565.

Allan, R. P. and G. E. Cook (1974). Thermospheric densities during an intense magnetic storm from the LOGACS experiment, J. Atmos. Terr. Phys., 36, 1739-1752.

Allen, S. J. and R. A. Vincent (1995). Gravity wave activity in the lower atmosphere: Seasonal and latitudinal variations, J. Geophys. Res., 100, 1327-1350.

Belikovich, V. V., E. A. Benediktov, N. P. Goncharov and A. V. Tolmacheva (1997). Diagnostics of the ionosphere and neutral atmosphere at E-region heights using artificial periodic inhomogeneities, J. Atmos. Terr. Phys., 59, 24472460.

Bilitza, D., K. Rawer, L. Bossy and T. Gulyaeva (1993). International reference ionosphere - past, present, future, Adv. Space Res., 13, 3-23.

Brekke, A. and Y. Kamide (1996). On the relationship between Joule and frictional heating in the polar ionosphere, J. Atmos, Terr. Phys., 58, 139-143.

Bristow, W. A., A. Greenwald and J. P. Villain (1996). On the seasonal dependence of medium-scale atmospheric gravity waves in the upper atmosphere at high latitudes, J. Geophys. Res., 101, 15685-15699.

Cole, K. D. (1975). Energy deposition in the thermosphere caused by solar wind, J. Atmos. Terr. Phys., 37, 939-949.

Coster, A and T. Tsugawa (2008). The impact of traveling ionospheric disturbances on global navigation satellite systemservices,http:/ / ursi-test.intec.ugent.be/files / URSIGA08/papers/FGp1.pdf

Fagundes, P. R., Y. Sahai, H. Takahashi, D. Gobbi and J. A. Bittencourt (1996). Thermospheric and mesospheric temperatures during geomagnetic storms at $23^{\circ} \mathrm{S}, \mathrm{J}$. Atmos. Terr. Phys., 58, 1963-1972.

Fritts, D. C. and M. J. Alexander (2003). Gravity wave dynamics and effects in the middle atmosphere, Rev. Geophys., 41, 1003, doi: 10.1029/2001RG000106.

Hanasz, J. H., F. De, R. Schreiber and M. Panchenko (2006). Pulsations of the auroral kilometric radiation, J. Geophys. Res., 111, A03209, doi:10.1029/ 2005JA011302.

Hernandez-Pajares, M, J. M. Juan and J. Sanz (2006). Medium -scale traveling ionospheric disturbances affecting GPS measurements: spatial and temporal analysis, J. Geophys. Res., 111, A07S11, doi:10.1029/2005JA011474.

Hocke, K. and K. Schlegel (1996). A review of atmospheric gravity waves and travelling ionospheric disturbances: 1982-1995, Ann. Geophysicae, 14, 917-940.

Istomin, Y. N. and T. B. Leyser (1997). Small-scale magnetic field-aligned density irregularities excited by a powerful electromagnetic wave, Phys. Plasmas, 4, 817-828.

Kersy, L and K. A. Hughes (1989). On the distinction between large-scale and medium-scale atmospheric gravity waves, Ann. Geophysicae, 7, 459-462.

Kirchengast, J. (1996). Elucidation of the physics of the gravity
wave-TID relationship with the aid of theoretical simulations, J. Geophys. Res., 101, 13353-13368.

Kotake, N., Y. Otsuka, T. Tsugawa, T. Ogawa and A. Saito (2006). Climatological study of GPS total electron content variations caused by medium-scale traveling ionospheric disturbances, J. Geophys. Res., 111, A04306, doi:10.1029/2005JA011418.

Millward, G. H., S. Quegar, R. J. Moffett, T. J. Fuller-Rowel and D. Rees (1993). A modeling study of the coupled ionospheric and thermospheric response to an enhanced high-latitude electric field event, Planet Space Sci., 41, 45-56.

Nicolls, M. J. and C. J. Heinselman (2007). Three-dimensional measurements of traveling ionospheric disturbances with the Poker Flat Incoherent Scatter Radar, Geophys. Res. Lett., 34, L21104, doi: 10.1029/2007GL031506.

Oliver, W. L., S. Fukao, Y. Yamamoto, T. Takami, M. D. Yamanaka, M. Yamamoto, T. Nakamura and T. Tsuda (1994). Middle and upper atmosphere radar observations of ionospheric density gradients produced by gravity wave packets, J. Geophys. Res., 99, 6321-6329.

Olson, W. P. and K. Moe (1974). Influence of precipitating charged particles on the high latitude thermosphere, J. Atmos. Terr. Phys., 36, 1715-1726.

Onoshi, T., T. Tsugawa, Y. Otsuka and J. J. Berthelier (2009). Ground based and satellite simultinous observations of medium scale travelling ionospheric disturbances, Geophys. Res. Abs., 11, EGU 2009-2236.

Prölss, G. W. (2006). Subauroral electron temperature enhancement in the nighttime ionosphere, Ann. Geophys., 24, 1871-1885.

Prölss , G. M. and M. Roemer (2000). Heating of the polar upper atmosphere, Phys. Chem. Earth., 25, 547-553.

Schlegel, K. (1982). Reduced effective recombination coefficient in the disturbed polar E-region, J. Atmos. Terr. Phys., 44, 183-185.

Yuan, Z., R. Fujii, S. Nozawa and Y. Ogawa (2005). Statistical height-dependent relative importance of the Lorentz force and Joule heating in generating atmospheric gravity waves in the auroral electrojets, J. Geophys. Res., 110, doi: 10.1029/2005JA011315.

Zesta, E., L. Lyons, C. P. Wang, E. Donovan, H. Frey and T. Nagai (2006). Auroral poleward boundary intensifications (PBIs): Their two-dimensional structure and associated dynamics in the plasma sheet, J. Geophys. Res., 111, A05201, doi:10.1029/ 2004JA010640.

\footnotetext{
${ }^{\star}$ Corresponding author. Dr. Syam Sundar De, Centre of Advanced Study in Radio Physics and Electronics, University of Calcutta, Kolkata 700 009, India; e-mail: de_syam_sundar@yahoo.co.in

(C) 2010 by the Istituto Nazionale di Geofisica e Vulcanologia. All rights reserved.
} 